\title{
Lesions of the Supramammillary Nucleus Decrease Self-Grooming Behavior of Rats Placed in an Open Field
}

\author{
Chiho K. Yamazaki, ${ }^{1}$ Tomoaki Shirao, ${ }^{1}$ Yoshio Sasagawa, ${ }^{1}$ \\ Yukio Maruyama, ${ }^{1}$ Hisanao Akita, ${ }^{2}$ Makoto Saji ${ }^{3}$ \\ and Yuko Sekino ${ }^{1,3}$
}

\begin{abstract}
Aim : Although subcortical regions send numerous efferent fibers to the hippocampus, their involvement in hippocampal functions has not been fully elucidated. The aim of this study was to determine the effect of the supramammillary nucleus (SuM) on the hippocampus. Methods : Neurons within the SuM of rats were destroyed by local injections of an excitotoxin, ibotenic acid, and the effects of the SuM-lesion on behaviors in an open field were investigated. Results : SuM lesions increased distance traveled, movement time and latency to start grooming, while they decreased time spent grooming. SuM lesions had no effect on rearing frequency or immobility time. Conclusion : Prolonged exploration and decrease in the total time spent grooming observed in the SuM-lesioned rats were consistent with the behavioral characteristics of hippocampal-lesioned rats of the previous reports, suggesting that the SuM is involved in the establishment of spatial memory by hippocampus during the initial exploration of a novel environment. In addition, the reduction of grooming in the SuM-lesioned animal suggests that SuM may be involved in emotion, such as anxiety. The results of this study show the involvement of the SuM in hippocampal function and in anxiety perceived in a novel environment. (Kitakanto Med J 2011 ; $61: 287 \sim 292)$
\end{abstract}

Key words : supramammillary nucleus, lesion, open field, exploration, grooming.

\section{Introduction}

Although subcortical regions send numerous efferent fibers to the hippocampus, their involvement in hippocampal functions has not been fully elucidated. It has been shown that activity of the supramammillary nucleus (SuM) in the hypothalamus is in synchrony with hippocampal theta rhythm, a slow rhythmic electroencephalogram related to hippocampal function, ${ }^{1,2}$ and that the SuM densely innervates the principal neurons in the CA2/CA3a region and the dentate gyrus (DG) of the hippocampus. ${ }^{3-6}$ In addition, pre-stimulation of the SuM facilitates population spikes in the hippocampus, ${ }^{7-9}$ and inhibition of neuronal activity in the SuM blocks the spread of limbic seizures in the hippocampus. ${ }^{10}$ These studies suggest that the SuM regulates signal processing in the hippocampus. ${ }^{5,11}$

To determine the effect of the SuM on the hippocampus, we destroyed neurons within the SuM of rats by local injections of an excitotoxin, ibotenic acid, and investigated effects of the SuM-lesion on hippocampal-related behaviors in an open field.

\section{Methods}

Adult male Wistar rats (nine-week old and weighing about 250g) were purchased from SLC (Hamamatsu, Japan), and were housed three to a cage (one for each group) in temperature-controlled $\left(22^{\circ} \mathrm{C}-25^{\circ} \mathrm{C}\right)$ conditions with a $12 \mathrm{hr}$ light/dark cycle (lights on at 8 : $00 \mathrm{hr}$ ). Food and water were freely available. Animals were handled daily for a few minutes until the

1 Department of Neurobiology and Behavior, Gunma University Graduate School of Medicine, 3-39-22 Showa-machi, Maebashi,

Gunma 371-8511, Japan 2 Department of Physiology, School of Allied Health Science, Kitasato University, 1-15-1 Kitasato, Sagamihara, Kanagawa 225-8555, Japan 3 Division of Pharmacology, National Institute of Health Sciences, 1-18-1 Kamiyoga, Setagaya-ku, Tokyo 158-8501, Japan

Received: March 23, 2011

Address: YUKO SEKINO Division of Pharmacology, National Institute of Health Sciences, 1-18-1 Kamiyoga, Setagaya-ku, Tokyo 158-8501 Japan 
day of the open field test. All experiments were performed in accordance with regulations outlined by Japanese law and NIH guidelines. All efforts were made to minimize the number of animals used and to minimize suffering.

Lesions were performed as previously described. ${ }^{10}$ In brief, rats were deeply anesthetized by intraperitoneal injection of sodium pentobarbital $(40 \mathrm{mg} / \mathrm{kg})$ (Nacalai, Kyoto, Japan), the skull was exposed by skin incision, and small holes were drilled to allow glass micropipette insertion. An ibotenic acid ( $\alpha-$ Amino- $^{-}$ 3-hydroxy-5-isoxazoleacetic acid) solution (10 mg/ ml) (Biomol, Plymouth Meeting, PA, USA) was freshly prepared before use and was mixed with wheatgerm agglutinin-conjugated horseradish peroxidase (HRPWGA, Honen, Tokyo, Japan) in order to verify sites of injections. Bilateral injections of the solution (100 $\mathrm{nl}$ each) into the SuM of the hypothalamus were made at the following coordinates (from bregma: anterior 4.7 $\mathrm{mm}$; lateral $\pm 0.2 \mathrm{~mm}$; ventral $8.0 \mathrm{~mm}$ ). The same volume of the vehicle was injected in sham operations. Before they recovered from anesthesia, rats were color marked with commercial products for dyeing human hair (Bigen hair dye powder, Hoyu Co., Ltd. Nagoya, Japan) on their backs. This allowed them to be detected by a video tracking system that traced their exploring behavior. The rats were put back into the cage after the operation and allowed to recover from the surgery. Open field tests were performed three days after the surgery. Six non-operated rats were also color marked under anesthetic three days before the experiment.

Open field tests were performed between 16:0018:00, under 240-lux illumination. A set of three rats, one each from operated (lesioned), sham-operated and non-operated groups, were tested on the same day. Each rat was placed gently and in random order into the center of the open field arena $\left(120 \mathrm{~cm}^{2}\right.$ floor with 40 $\mathrm{cm}$ high walls). Spontaneous behaviors of rats in the open field were videotaped for $30 \mathrm{~min}$ (WV-DR7, Sony Inc. Tokyo, Japan) with a DSP color camera (WAT-202D ; Watec. Co., Ltd. Yamagata, Japan) with a zoom lens (WAT-13VA286T; f2.8-6mm, F 1.2-64, auto iris, Watec. Co., Ltd.) mounted $2 \mathrm{~m}$ above the open field. The monitoring system was set in a separate room to avoid disturbing the animals. The floor of the open field was carefully cleaned with $70 \%$ ethanol at the end of each test.

The videotaped behaviors of the rats were analyzed after experiments by two observers blinded to the treatment group of the rats. Rearing was defined as the lifting of both forefeet off the floor and rearing up on its hindlimbs, regardless of on- or off-wall rearing; grooming was defined as the time spent for movements directed against self, which include forepaw movements over the body, scratching, licking, body gnawing, and face washing; immobility was defined as the time when there was no locomotion, including whisker or snout movements (i.e. standing, sitting, and lying position), for more than $3 \mathrm{sec}$ in each of the 5-min intervals. The distance traveled was automatically measured with a video tracking system, SMART (Panlab s.1., Barcelona, Spain). Movement time was calculated by subtraction of grooming and immobility times from the duration of the 5-min time blocks.

The behavioral scores of each 5-min block were statistically analyzed non-parametrically to avoid making assumptions about the normal distribution of the data. The Kruskal-Wallis one-way ANOVA was first performed to compare data of three groups of different treatments. When differences were found to be significant, non-operated and sham-operated groups were compared by a Mann-Whitney U-test. Only when this was not significant, lesioned rats were compared with the sham-operated groups by a MannWhitney U-test. Results are expressed here as means \pm SEM. A p-value of less than 0.05 was considered to be significant. All statistical analyses were carried out using StatView (SAS Institute Inc., Cary, NC, USA).

Upon completion of experiments, rats were deeply anesthetized with sodium pentobarbital $(50 \mathrm{mg} / \mathrm{kg})$ and were perfused transcardially with $0.9 \%$ saline containing heparin sodium (Aventis Pharma Ltd., Tokyo, Japan) $(2 \mathrm{U} / \mathrm{ml})$ at a rate of $37.5 \mathrm{ml} / \mathrm{min}$ for 4 min. Subsequently, $4 \%$ paraformaldehyde was perfused initially at a rate of $100 \mathrm{ml} / \mathrm{min}$ for $1 \mathrm{~min}$ and thereafter at a rate of $37.5 \mathrm{ml} / \mathrm{min}$. The brains were removed and post-fixed in the same fixative solution for $1 \mathrm{hr}$. After the brains were equilibrated in 30\% sucrose, they were frozen and sectioned coronally into $40 \mu \mathrm{m}$ slices with a sliding microtome. Sections were then incubated in $0.05 \%$ diaminobenzidine (DAB) and $0.003 \%$ hydrogen peroxide to visualize HRP-WGA. After the sections were placed on gelatin-coated slides, they were stained using thionin and examined under the light microscope to verify the locations of the lesions by ibotenic acid. The SuM was identified and delineated according to the criteria and maps of Paxinos and Watson. ${ }^{12}$ For diagrammatic reconstructions, the extent of each lesion was circumscribed on the map. One rat whose ibotenic acid injection failed to hit the SuM was not included in the statistical analysis. One sham-operated rat which had shivers and slow movements, and was found to have an intraventricular hemorrhage, was also excluded from the statistical analysis. 
A

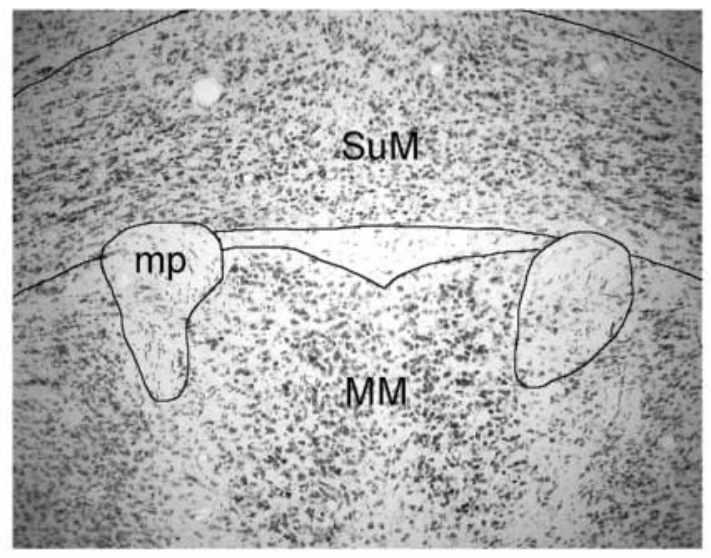

B

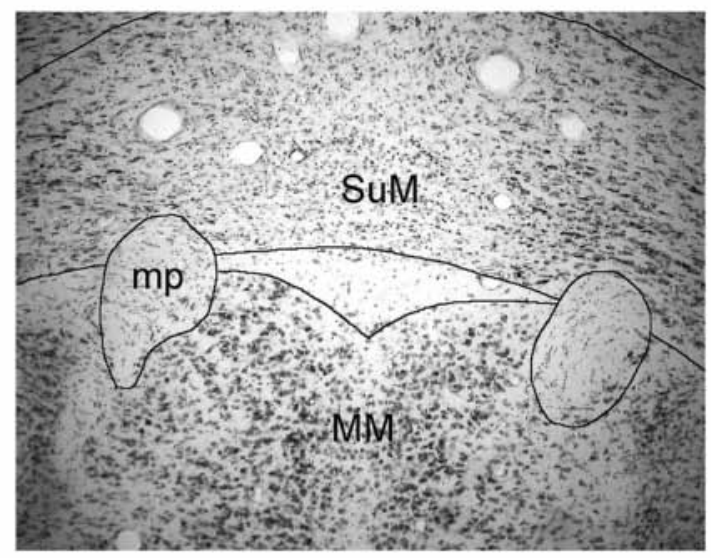

C
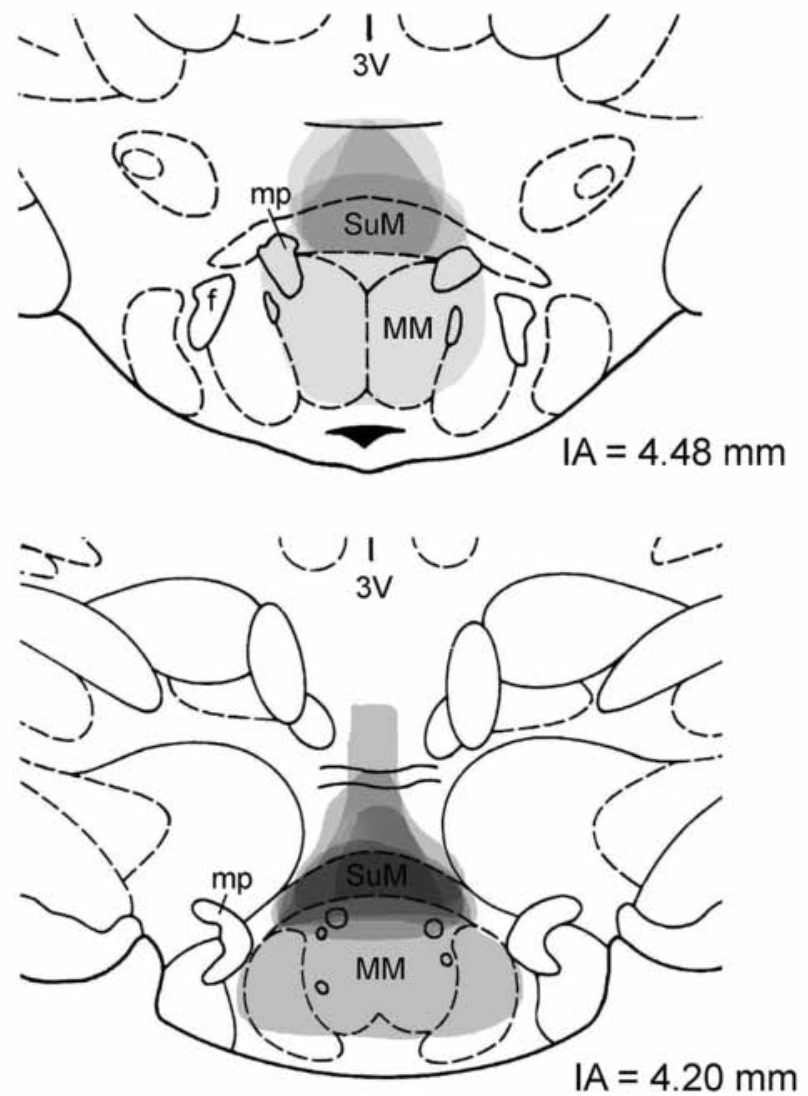

Fig. 1 The SuM-lesion by ibotenic acid. Representative Nissl staining sections showing intact cell bodies in the SuM of shamoperated rat (A) and replacement of neuronal cells with glial cells in SuM-lesioned rat (B) after ibotenic acid injection. C. Diagrammatic representation of the SuM-lesion. Each shaded area indicates extent of the lesion of each SuM-lesioned rat $(\mathrm{N}=$ 7). Superimposition of lesioned areas displays that the lesion is centered in the SuM. IA, interaural ; MM, medial mammillary nucleus ; mp, mammillary peduncle; SuM, supramammillary nucleus ; 3V, third ventricle.

\section{Results}

The lesion area was characterized by loss of neurons and increase in the number of glial cells (Fig. 1A, B). Areas of each lesion were indicated in gray for each case and overlaid on maps, shown in Fig. 1C. Although the size of lesion varied, the SuM was within the lesioned area for all rats. In sham-operated rats, no obvious alteration was found in SuM structure (Fig. 1A).

Behavioral analyses did not show any significant differences in rearing frequency (Fig. 2A) or in immobility time (Fig. 2B) between sham-operated and lesioned rats. But there were significant differences between these groups in movement time, distance traveled, grooming time (Fig. 2C, D, and E, respectively), and latency to start grooming (Fig. 3). The movement time of lesioned rats in 5-10 min blocks and in 15-20 min blocks was significantly longer than for shamoperated rats (Mann-Whitney $U$ test; $n=7, p<0.05$ ) (Fig. 2C). Lesioned rats traveled significantly longer than sham-operated rats in 15-20min blocks (MannWhitney U test; p<0.05) (Fig. 2D).

Time spent grooming within the first $5 \mathrm{~min}$ was significantly shorter for the lesioned group (16.0 \pm 19.8 s) than for the sham-operated control group (49.6士 $26.7 \mathrm{~s}) \quad($ Mann-Whitney $\mathrm{U}$-test; $\mathrm{p}<0.05)$. The grooming time of control groups peaked at the 5-10 min block and gradually decreased over the last three blocks. In contrast, the grooming time of lesioned rats remained at a similar level throughout the $30-\mathrm{min}$ testing period ; consequently, grooming time at the 5$10 \mathrm{~min}$ block was significantly shorter for these rats than for sham-operated rats (Mann-Whitney U-test ; lesioned rat $=34.4 \pm 16.6 \mathrm{~ms}$, sham-operated rat $=$ $109.6 \pm 71.2 \mathrm{~ms}, \mathrm{p}<0.01$ ) (Fig. $2 \mathrm{E}$ ). In addition, the lesioned group showed a significantly prolonged latency to start grooming compared to sham-operated rats (Mann-Whitney U-test; p<0.05) (Fig. 3). There were no significant differences for grooming time at the last three blocks between the three groups.

\section{Discussion}

The present study shows that lesion of the SuM increases the exploratory behaviors of rats placed in an open field. This result is consistent with results observed in rats with small lesions of the medial SuM. ${ }^{13}$ Prolonged exploration observed in the SuM-lesioned 
A

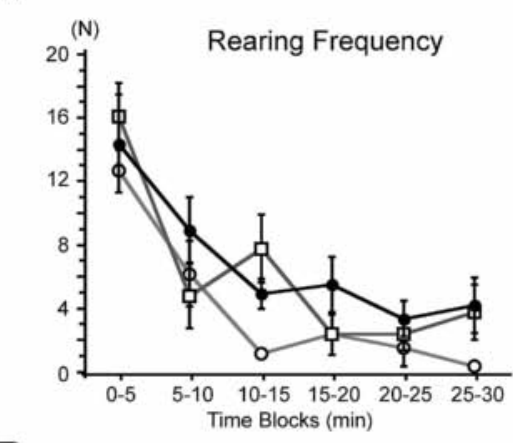

D

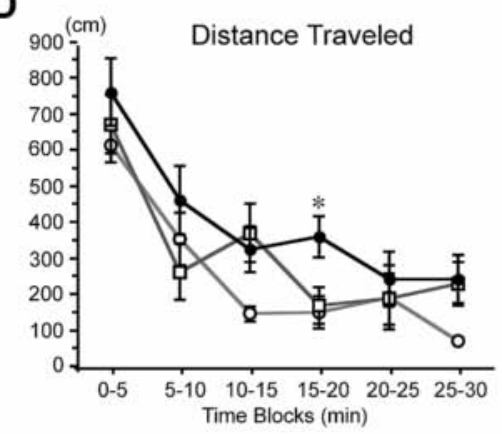

B

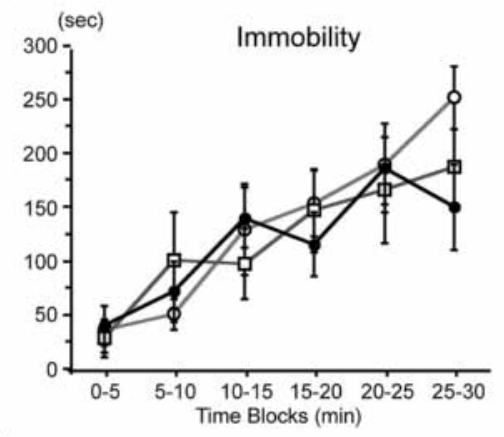

E

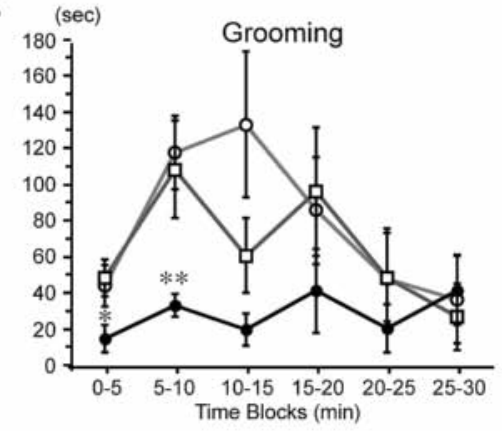

C

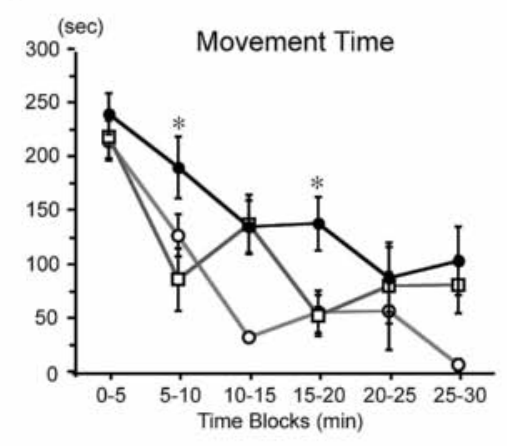

non-operated $(n=6)$

Sham-operated $(n=7)$

SuM-lesioned $(n=7)$

$* p<0.05, * * p<0.01$ Sham-operated vs SuM-lesioned

Fig. 2 The effects of SuM-lesion on behaviors of rats in an open field. Animals were placed in an open field for $30 \mathrm{~min}$. SuM lesioned rats exhibit more movement $(\mathrm{C}, \mathrm{D})$ and less grooming (E) compared to sham-operated and non-operated controls, but there was no significant differences in rearing frequency (A) and immobility (B). Behaviors of SuM-lesioned group was compared to Sham-operated control group only when there was no significant difference between two control groups (ie, non-operated and Sham-operated). Open circle, non-operated group; square, sham-operated group; filled circle, SuMlesioned group. ${ }^{*} \mathrm{p}<0.05,{ }^{* *} \mathrm{p}<0.01$, sham-operated versus SuM-lesioned by Mann-Whitney U-test. The bars indicate mean \pm SEM.

\section{Grooming Latency}

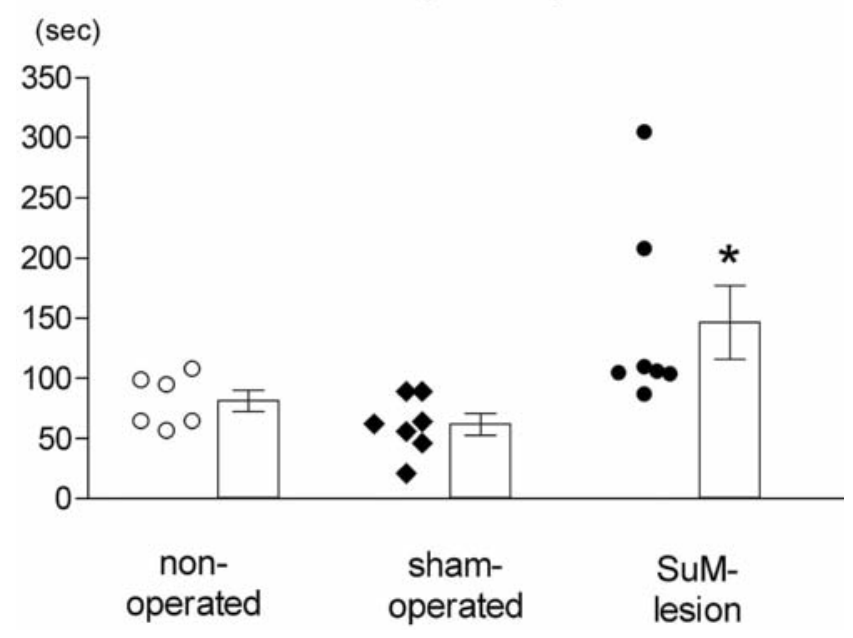

Fig. 3 Scatter plots of the latencies taken by the individual animal of each experimental group to initiate grooming during 30-min open field test. The bars indicate mean \pm SEM of grooming latency of each group. SuM-lesioned rats showed significantly prolonged grooming latency compared to sham-operated group $\left({ }^{*} \mathrm{p}<0.05\right.$, MannWhitney U-test). There was no significant difference in the mean grooming latency between non-operated and sham-operated groups. rats in an open field can be interpreted as a deficiency in the establishment of spatial memory, because spatial memory is thought to be established during the initial exploration of a novel environment. ${ }^{14}$ Rats with hippocampal lesions show similar prolonged exploration $^{15-18}$ and impairment of spatial memory. ${ }^{16,19-23}$ Thus, the SuM apparently is involved in hippocampal memory function.

A novel finding of the present study is that SuM lesions decrease the total time spent grooming and increase the latency to the initial grooming behavior. Grooming behavior can be observed after a variety of activities. $^{24}$ In an open field test, it is seen after exploration of a novel environment. ${ }^{25,26}$ Upon placement in an open field, rats explore to acquire information about the new environment and grooming begins with some latency. Once the environment is no longer novel and the rats are settled in their surroundings, the exploratory and grooming behaviors return to the normal level. Thus, an increase in grooming latency seems to parallel the prolonged exploratory behavior. A reduction in grooming behavior is also observed in rats with kainic acid lesions in the hippocampus. ${ }^{18}$ This indicates that lesion of the SuM has similar effects to the lesion in the hippocampus, further supporting the idea that the SuM is involved in hippocampal 
memory function. However, this cannot explain the observed decrease in total grooming activity in the SuM lesioned rats. If the SuM lesion impairs spatial memory formation alone, subsequent grooming should be normal and an increase in grooming time should be observed once the initial exploring behavior is over. In the SuM lesioned rats, grooming time remained very low throughout the $30 \mathrm{~min}$ testing period. This suggests that there must be other factor(s) that cause attenuation of grooming.

One possible implication for the reduction of grooming in the SuM-lesioned animal is that the SuM may be involved in emotion, such as anxiety. It has been reported that expression of $\mathrm{c}-\mathrm{fos}$, a marker of increased neuronal activity, is increased in the SuM when rats are placed in a novel environment, ${ }^{27,28}$ while a low dose of an anxiolytic drug, benzodiazepine, suppresses $\mathrm{c}$-fos expression in the SuM. ${ }^{29}$ Also, cholecystokinin (CCK) immunopositive neurons are known to induce anxiogenic responses ${ }^{30}$ and such neurons are found in the SuM. ${ }^{31,32}$ These observations are in consistent with the idea that the SuM participates in emotion. It is interesting to note that CCK is also known to increase the grooming behavior of mice placed in a novel environment. ${ }^{33}$ Besides affecting anxiety, lesion of the SuM may alter the CCK system, which in turn could attenuate grooming behavior in the lesioned rats.

In conclusion, the present study suggests that SuM activity is involved in anxiety perceived in a novel environment as well as in the acquisition and storage of spatial information performed in the hippocampus.

\section{Acknowledgements}

This work was supported by Grants-in-Aid for Scientific Research from the Ministry of Education, Culture, Sports, Science and Technology of Japan (19200029) and CREST, JST. We thank Dr. Shizuka Kobayashi for the technical assistance with the SuM -lesion surgery.

\section{References}

1. Kirk IJ and McNaughton N. Mapping the differential effects of procaine on frequency and amplitude of reticularly elicited hippocampal rhythmical slow activity. Hippocampus 1993 ; 3(4) : 517-525.

2. Kocsis B and Vertes RP. Characterization of neurons of the supramammillary nucleus and mammillary body that discharge rhythmically with the hippocampal theta rhythm in the rat. J Neurosci 1994 ; 14 (11 Pt 2): 7040-7052.

3. Haglund L, Swanson LW and Kohler C. The projection of the supramammillary nucleus to the hippocampal formation: an immunohistochemical and anterograde transport study with the lectin PHA-L in the rat. J Comp Neurol 1984 ; 229(2): 171-185.

4. Magloczky Z, Acsady L and Freund TF. Principal cells are the postsynaptic targets of supramammillary afferents in the hippocampus of the rat. Hippocampus $1994 ; 4(3)$ : 322-334.

5. Ochiishi T, Saitoh Y, Yukawa A, et al. High level of adenosine A1 receptor-like immunoreactivity in the CA2/ CA3a region of the adult rat hippocampus. Neuroscience 1999; 93(3) : 955-967.

6. Vertes RP. PHA-L analysis of projections from the supramammillary nucleus in the rat. J Comp Neurol 1992 ; 326(4) : 595-622.

7. Carre GP and Harley CW. Population spike facilitation in the dentate gyrus following glutamate to the lateral supramammillary nucleus. Brain Res 1991；568(1-2) : 307310.

8. Mizumori SJ, McNaughton BL and Barnes CA. A comparison of supramammillary and medial septal influences on hippocampal field potentials and single-unit activity. J Neurophysiol 1989; 61(1): 15-31.

9. Nakanishi K, Saito H and Abe K. The supramammillary nucleus contributes to associative EPSP-spike potentiation in the rat dentate gyrus in vivo. Eur J Neurosci 2001; 13(4) : 793-800.

10. Saji M, Kobayashi S, Ohno K, et al. Interruption of supramammillohippocampal afferents prevents the genesis and spread of limbic seizures in the hippocampus via a disinhibition mechanism. Neuroscience $2000 ; 97(3): 437-445$.

11. Sekino $\mathrm{Y}$, Obata K, Tanifuji M, et al. Delayed signal propagation via $\mathrm{CA} 2$ in rat hippocampal slices revealed by optical recording. J Neurophysiol 1997; 78(3) : 16621668.

12. Paxinos $\mathrm{G}$ and Watson $\mathrm{C}$. The rat brain in stereotaxic coordinates. Sandiego: Academic Press, 1998.

13. Pan WX and McNaughton N. The role of the medial supramammillary nucleus in the control of hippocampal theta activity and behaviour in rats. Eur $\mathbf{J}$ Neurosci 2002 ; 16(9) : 1797-1809.

14. Wilson MA and McNaughton BL. Dynamics of the hippocampal ensemble code for space. Science 1993; 261(5124) : 1055-1058.

15. Gray JA and McNaughton N. Comparison between the behavioural effects of septal and hippocampal lesions: a review. Neurosci Biobehav Rev 1983; 7(2): 119-188.

16. Handelmann GE and Olton DS. Spatial memory following damage to hippocampal CA3 pyramidal cells with kainic acid: impairment and recovery with preoperative training. Brain Res $1981 ; 217(1): 41-58$.

17. Jarrard LE. Behavior of hippocampal lesioned rats in home cage and novel situation. Physiology \& Behavior $1968 ; 3(1): 65-70$.

18. Nyakas C, De Kloet ER, Veldhuis HD, et al. Hippocampal corticosterone receptors and novelty-induced behavioral activity: effect of kainic acid lesion in the hippocampus. Brain Res 1983; 288(1-2) : 219-228.

19. Bannerman DM, Yee BK, Good MA, et al. Double dissociation of function within the hippocampus : a comparison of dorsal, ventral, and complete hippocampal cytotoxic lesions. Behav Neurosci 1999; 113(6) : 1170-1188.

20. Cassel JC, Cassel S, Galani R, et al. Fimbria-fornix vs selective hippocampal lesions in rats: effects on locomotor activity and spatial learning and memory. Neurobiol Learn Mem 1998; 69(1) : 22-45.

21. Jarrard LE. On the role of the hippocampus in learning and memory in the rat. Behav Neural Biol 1993; 60(1): $9-26$. 
22. Morris RG, Garrud P, Rawlins JN, et al. Place navigation impaired in rats with hippocampal lesions. Nature 1982; 297(5868): 681-683.

23. Olton DS, Becker JT and Handelmann E. Hippocampus, space and memory. Behav Brain Sci 1979; 2: 313-366.

24. Spruijt BM, van Hooff JA and Gispen WH. Ethology and neurobiology of grooming behavior. Physiol Rev 1992 ; $72(3): 825-852$.

25. Eilam D and Golani I. Home base behavior of rats (Rattus norvegicus) exploring a novel environment. Behav Brain Res $1989 ;$ 34(3): 199-211.

26. van Erp AM, Kruk MR, Meelis W, et al. Effect of environmental stressors on time course, variability and form of self-grooming in the rat: handling, social contact, defeat, novelty, restraint and fur moistening. Behav Brain Res $1994 ; 65(1): 47-55$.

27. Ito M, Doya K, Shirao T, et al. Spatial distribution of Fos-positive neurons in the supramammillary nucleus and the hippocampus of rats placed in a novel enviroment. Soc. Neurosci. Abstr. 2003; 717: 13.

28. Wirtshafter D, Stratford TR and Shim I. Placement in a novel environment induces fos-like immunoreactivity in supramammillary cells projecting to the hippocampus and midbrain. Brain Res 1998; 789(2) : 331-334.

29. Beck $\mathrm{CH}$ and Fibiger HC. Conditioned fear-induced changes in behavior and in the expression of the immediate early gene c-fos: with and without diazepam pretreatment. J Neurosci 1995; 15(1 Pt 2): 709-720.

30. Harro J, Vasar E and Bradwejn J. CCK in animal and human research on anxiety. Trends Pharmacol Sci 1993; 14(6) : 244-249.

31. Kiss J, Csaki A, Bokor H, et al. Topographic localization of calretinin, calbindin, VIP, substance P, CCK and metabotropic glutamate receptor immunoreactive neurons in the supramammillary and related areas of the rat. Neurobiology (Bp) 1997 ; 5(3) : 361-388.

32. Kiyama H, Shiosaka S, Tateishi K, et al. Cholecystokinin8-like immunoreactive neuron pathway from the supramammillary region to the ventral tegmental nucleus of Gudden of the rat. Brain Res $1984 ; 304(2): 397-400$.

33. Dunn AJ, Guild AL, Kramarcy NR, et al. Benzodiazepines decrease grooming in response to novelty but not ACTH or beta-endorphin. Pharmacol Biochem Behav $1981 ; 15(4)$ : 605-608. 